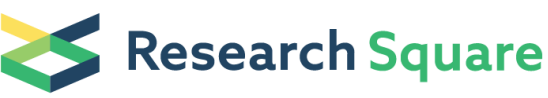 \\ Preprints are preliminary reports that have not undergone peer review. \\ They should not be considered conclusive, used to inform clinical practice, or referenced by the media as validated information.
}

\section{No impact of psychosocial stress on glioblastoma development}

\section{Quentin Durrechou}

CHU Bordeaux Pôle Cancérologie: Centre Hospitalier Universitaire de Bordeaux Pole Cancerologie

\section{Julien Engelhardt}

CHU Bordeaux GH Pellegrin: Centre Hospitalier Universitaire de Bordeaux Groupe hospitalier Pellegrin

\section{Vincent Jecko}

CHU Bordeaux GH Pellegrin: Centre Hospitalier Universitaire de Bordeaux Groupe hospitalier Pellegrin

\section{Charlotte Domblides}

CHU Bordeaux Pôle Cancérologie: Centre Hospitalier Universitaire de Bordeaux Pole Cancerologie

\section{Isabelle Catry-Thomas}

CHU Bordeaux Pôle Cancérologie: Centre Hospitalier Universitaire de Bordeaux Pole Cancerologie

\section{Hugues Loiseau}

CHU Bordeaux GH Pellegrin: Centre Hospitalier Universitaire de Bordeaux Groupe hospitalier Pellegrin

\section{Charlotte Planchon}

CHU Bordeaux GH Pellegrin: Centre Hospitalier Universitaire de Bordeaux Groupe hospitalier Pellegrin

\section{Sandrine De Grimal}

CHU Bordeaux GH Pellegrin: Centre Hospitalier Universitaire de Bordeaux Groupe hospitalier Pellegrin Charlotte BRONNIMANN ( $\nabla$ charlotte.bronnimann@chu-bordeaux.fr )

Hopital St André https://orcid.org/0000-0002-2208-0545

\section{Research Article}

Keywords: psychosocial stress, glioblastoma, stressors, stress level

Posted Date: January 25th, 2022

DOI: https://doi.org/10.21203/rs.3.rs-1138824/v2

License: (c) (i) This work is licensed under a Creative Commons Attribution 4.0 International License.

Read Full License 


\section{Abstract}

No impact of psychosocial stress on development of glioblastoma

Purpose: Psychosocial stress represents an important source of questions in the potential implication of origin of cancer. The aim of the study was to assess stress prevalence prior to diagnosis of wild-type IDH glioblastoma.

Methods: This prospective single-center study enrolled consecutive new cases of wild-type IDH glioblastoma diagnosed between December 2019 and March 2021 at the University Hospital of Bordeaux. A standardized patient self-assessment stress questionnaire explored both the presence of stressor exposure and the intensity of patient stress level prior to the diagnosis. Four groups were included: high stressors/high stress, high stressors/low stress, low stressors/low stress, and low stressors/high stress. Patient characteristics were collected. Statistical analysis was based on the Chisquare test and the Kaplan-Meier survival estimator.

Results: Sixty-four patients with a median age of 66 years were included. Glioblastoma involved predominantly the frontal lobe (39\%). Thirty-six patients $(56.3 \%)$ presented a low stressor/low stress profile. Stress corresponded mainly to the death of a loved one or to family health problems. Among working professionals, 20 patients $(67.5 \%)$ reported low-intensity work stress. A history of depression was found in $30 \%$. Progression-free survival at 6 months was $45.3 \%$ and median overall survival was estimated to be 16.5 months. Level and presence of stress did not differ based on location of tumour. No association was found between stress and tumour progression or overall survival.

Conclusion: A majority of patients in this study had low exposure to stressors as well as low stress level. Psychological stress did not seem to favour the emergence of glioblastoma or survival.

\section{Introduction}

Stress corresponds to the psychological, physical and emotional reaction that a subject can develop to cope with situations and pressures imposed on them. [1] By activating the adrenergic system and the cortisol pathway, stress becomes responsible for physiological changes (increase in blood pressure, acceleration of the heart rate, disruption of circadian rhythms, etc.) and the hypothesis of an association between stress and increased health risk emerged many years ago. [2] The impact of stress on increased risk of morbidity and mortality is now well established. [3,4] Different types of stressful events ("stressors") have been identified (work, bereavement, trauma, financial difficulties, etc.) and can be spread over a very variable time scale. [5] Stress is now well identified as a risk factor, particularly in the context of cardiovascular diseases [6], but its implication in many other medical fields remains unclear. In oncology, the implication of stress on oncogenesis and tumour progression remains a source of questions. A strong association between stress and cancer has not clearly been established based on data available in the literature. [7-9] In primary central nervous tumours, few risk factors have been identified. $[10,11]$ The potential presence of an identified psychosocial stress prior to diagnosis represents 
an important source of routine questions for both patients and caregivers. To our knowledge, no study has yet evaluated the impact of acute or chronic stress on the incidence of brain tumours.

The study aimed to assess the prevalence of exposure to acute and chronic psychosocial stress, and the response of patient to the stress in a consecutive cohort of newly diagnosed patients with wild-type IDH glioblastoma at the University Hospital of Bordeaux using a standardized self-assessment scale. [12] Secondary objectives were to analyze the potential association between stress and glioblastoma progression and survival.

\section{Methods}

Adult patients included were patients with newly diagnosed wild-type IDH glioblastoma and histologically confirmed from December 2019 to March 2021 at the University Hospital of Bordeaux. The study was prospective. Follow-up ended at death or until last news. Exclusion criteria were the presence of a mutant IDH glioblastoma and the presence of advanced cognitive disorders incompatible with filling in the questionnaire. Due to the Covid-19 health crisis during 2020 (confinement, teleworking, telephone announcement), and some cases of intense psychological suffering at the announcement of the diagnosis, some eligible patients could not complete the questionnaire and were therefore not included in the present study. All patients included signed an informed consent form.

After the announcement of the diagnosis, the patient received the stress questionnaire (Appendix 1) from the specialist nurse or the neuro-oncologist separated into two different parts. The Cungi scale used in our study has been validated and recognized as a sensitive and reproducible scale. The stress questionnaire completed by patients was developed in 1997 by French psychiatrist Charly Cungi, specialist in cognitive and behavioral therapy, and author of numerous studies about stress. Thanks to the multiple dimensions explored as well as its sensitivity, reproducibility and reliability, this questionnaire constitutes a recognized and validated scale. The first part of the questionnaire entitled "Brief Rating Scale for Stressors" includes 8 questions and the answers to each question are calibrated with a score from 1 to 6 (from "no" to "extremely"). The patient then related the potential history of psychological trauma experienced (any time in their life) as well as their exposure to stressors (health, family, loss, sentimental, financial, and professional or other stressors). If recent trauma was identified in the two years prior to diagnosis, the patient was asked to provide written details of this trauma. After adding points obtained for each question, a global score was calculated and it was then possible to define a stressor intensity level (high level of stressor exposure if score above 18). Question 8 of this part (Do I have a disease? Rate its severity and the discomfort for you) was analyzed independently from the rest of the questionnaire and new intervals were recalculated. Indeed, the stress induced by announcement of the diagnosis would have been responsible for a significant bias in this question. The second part of the questionnaire entitled "Brief stress rating scale" included 11 questions about how the stress was expressed through character traits, emotivity, potential psychosomatic manifestations as well as feelings about their health. After adding points obtained for each question, a global score was calculated and then it was possible to define a stress intensity level (high level of stress if score above 30). After calculating 
the overall scores, we differentiated the patients into 4 different categories according to their level of stressor exposure and stress response (category 1: high stressors /high stress; category 2: high stressors / low stress; category 3: low stressors / low stress; category 4: low stressors / high stress). Patient characteristics included clinical, radiological, oncological management and follow-up. Habits reported included excessive alcohol consumption or smoking over 10 pack-years. A history of depression corresponded to an active or past depression for major depression of DSM criteria. Multifocal location corresponded to a presentation with at least 2 consecutive lobes involved with tumoral contiguity. Tumour characteristics included neuropathology according to the $2016 \mathrm{WHO}$ classification of brain tumours and methylation status of the MGMT promoter. Time of first symptoms, time of diagnosis corresponding to surgery and time of acute stress, if present, were reported.

Patient characteristics as well as progression free survival (PFS) and overall survival (OS) were analyzed using a Chi-square test. The Kaplan Meier estimator and the Log Rank test enabled the development and comparison of survival curves. Subgroups analyses made it possible to calculate the progression and survival rates according to several parameters (stressor level, stress level, stressor / stress categories, traumatic history etc).

\section{Results}

Between December 2019 and March 2021, 65 patients were included in this study. One patient was excluded due to IDH mutant status. Median age of patients was 66 years, with a majority of male patients (sex ratio 1.2). Patient characteristic are presented in Table 1. Glioblastoma mainly involved the frontal lobe (25 patients). The methylation status of MGMT promoter was found in 20 cases among the 50 cases assessed. Patients with professional activity at diagnosis represented 30 patients $(47 \%)$ of the cohort. A history of chronic disease at diagnosis was reported in 46 patients with a majority of high blood pressure in 26 patients, dyslipidemia in 17 patients, diabetes in 11 patients and coronary disease in 10 patients. A history of depression was reported for 19 patients (30\%) and 12 patients (19\%) were taking psychotropic medication at the time of diagnosis (7 patients with anxiolytic treatment such as benzodiazepines and 5 with antidepressant treatment). Seventeen percent (11 patients) of the cohort were taking a beta-blocker treatment for cardiovascular disease at diagnosis. Clinical presentation was mainly a neurological deficit in 33 patients, and performance status was less or equal to 1 in 45 patients. Thirty patients (47\%) underwent a brain biopsy while 34 patients $(53 \%)$ had a surgical resection including $9(26 \%)$ with complete resection and 25 (74\%) subtotal resection. Four patients with complete resection had a Carmustine wafer implantation during surgery. After a multidisciplinary meeting, a concomitant radio-chemotherapy was the oncological treatment proposed for 57 patients (89\%). Treatment with Temodal alone was decided for 6 patients (9\%). One patient underwent palliative care after surgery.

After category recombination, a majority of patients (36 patients $-56.3 \%$ ) presented a "low stressor / low stress" profile (Table 1). According to the first question regarding the experience of psychological trauma, 33 patients (52\%) reported low or absence of exposure to psychological trauma (score 1 to 3 ). The loss of a loved one accounted for $80 \%$ of the psychological traumas described by patients. Forty patients (63\%) 
reported low or no acute stress in the 2 years prior to diagnosis and the existence of extreme acute stress was found in only 10 patients $(15,6 \%)$. Family health problems were the most represented category of stressful events (31\%) followed by the loss of a family member $(27.5 \%)$ and job strain $(27.5 \%)$. Divorces and other unclassified stressful events each accounted for $7 \%$ of stressful events. Among all patients, 37 patients $(57.8 \%)$ did not mention any job strain and among the 30 working patients, $20(67.5 \%)$ reported low job strain. After analyzing all the results of the first part of the questionnaire, a large majority of patients $(67 \%)$ had a low or very low level of stressors. Regarding question 8 of the first part of the questionnaire, 39 patients $(62.9 \%)$ considered the diagnosis of glioblastoma with a significant or extreme severity. Similarly, the second part of the questionnaire showed that 44 patients (69\%) had a low or very low level of stress.

For patients on beta-blocker medication, the stress level was low or very low in $73 \%$ of cases but the difference was not significant $(p=0,754)$. Patients with frontal tumour location had a non-significant increase in stress level ( $40 \%$ vs $21 \% ; p=0,121)$ and depression rate did not appear to be affected by tumour location ( $p=0,973)$. There was no significant difference in stress between right and left hemisphere tumour, nor between dominant and non-dominant hemisphere $(p=0,450)$. Furthermore, there appeared to be no significant difference between men and women regarding the level of exposure to stressors (including job strain) $(p=0,184)$ or the level of stress $(p=0,112)$. Based on these results, exposure to stressors as well as the intensity of the stress level appear to be relatively low in the glioblastoma population.

Median time between reported stress, if present, and diagnosis was 11 months (Figure 1). Among all the patients in this study and during the first 6 months of follow-up, 35 patients $(54,7 \%)$ presented a tumoral progression according to defined progression criteria (typical radiological progression or pseudoprogression with clinical degradation, new line of treatment, palliative care or death). Therefore, the 6 month PFS was $45,3 \%$. No significant association was demonstrated between tumour progression, overall survival and exposure to stressors or stress level. Median OS was 16.5 months. History of depression $(p=0,755)$, use of beta-blockers $(p=0,683)$ and MGMT status $(p=0,309)$ did not appear to significantly impact overall survival. The multifocal nature of the tumour was the only parameter that significantly impacted progression $(p=0,015)$ and survival $(p=0,002)$ negatively.

\section{Discussion}

The present study described for the first time stressor exposure for patients with glioblastoma at diagnosis and stress response in this population. A majority of patients did not report acute stress prior to diagnosis of glioblastoma nor a high level of stress. PFS and OS were similar in the different categories of stress response. The only factor that significantly and negatively affected PFS and OS in this study was the multifocal location.

Although stress represents an ambivalent response that can be beneficial in certain situations (concept of "fight or flight response") [13], the association between stress and pathologies are numerous [14] and concern almost all medical specialties (depression [15], metabolic syndrome, coronary artery disease [16], 
psoriasis [17], inflammatory bowel disease [18] etc,). Most epidemiological studies affirm that the prolonged perception of a high level of stress remains associated with reduced physical and mental condition. $[19,20]$ This data is consistent with our results which find the higher rate of depression in the "high stressors / high stress" category of patients.

After much fundamental and applied research, a physiological and molecular rationale has been described in the association between stress and cancer. The autonomic nervous system and the hypothalamic-pituitary-adrenal system are thought to be responsible for the majority of interactions between stress and cancer via the secretion of catecholamines and cortisol. [21-23] Inflammation and a decrease in immunoprotective functions induced by the secretion of cortisol and catecholamines could explain the mechanisms leading to tumour promotion and progression. [21,24] In several publications, stress-related psychosocial factors have been suggested to contribute to incidence of cancer, tumour progression and worst survival in cancer patients $[9,25]$ but most studies have focused on high-incidence cancer (especially in breast cancer [26-28] ). Despite all the studies carried out, the stress-cancer association is currently not very robust. However, primary brain cancer incidence remains low (almost 20/100,000 person-years in France [29] ) and the risk factors for glioblastoma (brain radiation, genetic predisposition) are rarely found in medical practice. The multiple questions from patients about the origin of the disease, led us to carry out this study and, to our knowledge, this is the first study to assess the impact of stress on the emergence of glioblastomas.

To address this question, we selected patients with primary brain tumours at the time of diagnosis to break free from stress induced by cancer management. Due to the Covid-19 health crisis during 2020, several eligible patients could not be included in our study. Furthermore, the study did not include cognitive test evaluation especially for patients excluded due to advanced cognitive disorder, incompatible with filling in the questionnaire. Moreover, we choose glioblastoma with a wild-type IDH profile, an aggressive tumour recognized for its rapid growth and patient symptoms briefly preceding diagnosis. This choice made it possible to limit the risk of patients presenting symptom-induced stress directly through the slow and chronic evolution of infiltrative gliomas with IDH mutation. [30] The results of this study indicated a majority of men in the glioblastoma population with a median age of 66 years old and this data is almost identical to the characteristics found in the literature. [11] Both in our study and in the literature, MGMT promoter methylation was identified in $40 \%$ of cases. [31] Genetic susceptibility represents approximately $5 \%$ of glioblastomas [11] and a family history of brain tumours was found in 4 patients $(6 \%$ ) in our study but no details were available regarding the histological type. Cardiovascular risk factors or coronaropathy represented a majority $(56 \%)$ of medical histories of patients and these pathologies are known for their high-prevalence in the stressed population. [6,32,33] The depression rate found in this study (30\%) was a little bit higher than in the general population (20\%) [34] and is probably explained by the cognitive impairment in the glioblastoma population. The interaction of beta-blockers with the adrenergic pathway involved in stress and depression has been the subject of many publications concerning their impact on these pathologies [35-38] and the proportion of patients treated with beta-blockers in our study seems similar to the proportion observed in the general population (20\%). [39] Location of the tumour did not impact stress, especially frontal location that can 
induce hypersensitivity which can lead to organic depression and anosodiaphoria which can lead to emotional indifference. The frontal lobe is involved in several functions including emotional regulation and a large portion of the patients in this study (up to $61,5 \%$ in the high stressor / high stress category) had an extensive frontal lobe tumour. This could have made interpretation of the results more difficult, but no significant association was found between frontal location and stress level. In addition, these results are consistent because the frontal lobe represents the largest lobe of the brain and is one of the brain regions most affected by gliomas. [40]

Some studies have described a better quality of life in cases of minor hemisphere syndrome. [41] The dominant hemisphere could be defined for all patients, however in our study there did not appear to be a significant difference in stress between left and right hemisphere tumours. A large proportion of patients with recent diagnosis of glioblastoma reported no exposure to acute stress in the two years preceding the diagnosis. More than half of patients (56.3\%) presented a "low stressor / low stress" profile and these results suggest an unlikely association between stress and glioblastoma incidence. It seems important to emphasize that the "low stressor / low stress" patients corresponded to the oldest patients (pensioners). The proportion of job strain found in this study is low and the publications about job strain do not find a significant increase in risk of cancer. [42,43] Among the patients who identified recent acute stress, the time between the traumatic event and the first symptom was 11 months. In this single-center study with a socioculturally homogeneous population, health problems, loss of a loved one or job strain were the main stressors and certain types of stressors may have been underestimated (financial, healthcare access, war, etc.). The results of the phase III EORTC-NCIC trial (STUPP protocol for high grade gliomas) published in 2004 found a median OS of 14.6 months and a median PFS of 6.9 months for patients treated with radiotherapy and Temodal. [44] In our study, tumour progression seemed to be greater (PFS at 6 months = $45.3 \%$ ), probably explained by the presence of more serious patients, sometimes treated with Temodal alone or palliative care only. Tumour progression and overall survival do not seem to be influenced by the existence or intensity of the patient's stress. The multifocal nature of the tumour was the only characteristic that negatively impacted progression and survival in this study. Several biases and limitations are present in this single-center study with a small number of patients. An important limit is the absence of a control cohort and therefore the lack of possibility of calculating the hazard ratio on stress exposure. In addition, the retrospective and declarative nature of the questionnaires may overestimate the stress level in the context of recent diagnosis of cancer. On the other hand, talking about stress and cancer could tend to relativize patient stress in comparison to the diagnosis of cancer and then minimize the presence of real stress that might have been important in another context. The cognitive distortion after receiving the diagnosis and the cognitive impairment induced by the tumour are probably responsible for a significant recall bias. Despite the inter and intra individual variability of stress as well as the multiple methodological difficulties to objectively evaluate this qualitative variable [45], several evaluation scales have been described for stressors [45-47] and stress level [48] and the Cungi scale used in our study has been validated and recognized as a sensitive and reproducible scale. [12] The stress questionnaire completed by patients was developed in 1997 by the French psychiatrist Charly Cungi, specialist in cognitive and behavioral therapy, and author of numerous studies about stress. Thanks to the multiple dimensions explored as well as its sensitivity, reproducibility and reliability, this 
questionnaire constitutes a recognized and validated scale. Although some studies report a reduction in the risk of death from cancer, with no benefit in overall survival $[21,49,50]$ the personalized and multimodal management of the stress of our patients remains essential in order to guarantee the best possible quality of life. The survival benefit of stress management as well as the stress impact on efficacy of immunotherapy are other avenues worth exploring.

\section{Conclusion}

Although stress is an essential concern for patients with a primary brain tumour, psychological stress does not appear to impact the emergence of brain tumours nor the survival of patients with glioblastoma.

\section{Declarations}

not applicable

Funding: not applicable

Conflicts of interest: the authors declare no conflict of interest

Code availability: not applicable

Ethics approval: study was declared in $\mathrm{CHU}$ of Bordeaux ethic committee

Consent to participate: all patient agreed to participate and signed an informed consent

Consent for publication: all the author consented for publication

Contribution of the authors: all authors corrected the manuscript. Dr Durrechou and Dr Bronnimann worked on the datas, realized the statistics and wrote the manuscript. Ms De-Grimal, Dr Catry-Thomas and Dr Bronnimann recovered data from patients.

COI : not applicable

Data are in depository

\section{References}

1. Levav I, Rutz W. The WHO World Health Report 2001 new understanding--new hope. Isr J Psychiatry Relat Sci. 2002;39(1):50-56

2. Segerstrom SC, Miller GE. Psychological Stress and the Human Immune System: A Meta-Analytic Study of 30 Years of Inquiry. Psychological Bulletin. 2004;130(4):601- 630

3. Rahe RH, Meyer M, Smith M, Kjaer G, Holmes TH. Social stress and illness onset. Journal of Psychosomatic Research. 1964;8(1):35-44 
4. Dhabhar FS. Effects of stress on immune function: the good, the bad, and the beautiful. Immunol Res. 2014;58(2-3):193-210

5. Turner RJ, Wheaton B, Lloyd DA. The Epidemiology of Social Stress. American Sociological Review. 1995;60(1):104

6. Steptoe A, Kivimäki M. Stress and Cardiovascular Disease: An Update on Current Knowledge. Annu Rev Public Health. 2013;34(1):337-354

7. Lin $Y$, Wang C, Zhong Y, et al. Striking life events associated with primary breast cancer susceptibility in women: a meta-analysis study. J Exp Clin Cancer Res. 2013;32(1):53

8. Schoemaker MJ, Jones ME, Wright LB, et al. Psychological stress, adverse life events and breast cancer incidence: a cohort investigation in 106,000 women in the United Kingdom. Breast Cancer Res. 2016;18(1):72

9. Chida Y, Hamer M, Wardle J, Steptoe A. Do stress-related psychosocial factors contribute to cancer incidence and survival? Nat Rev Clin Oncol. 2008;5(8):466-475

10. Ostrom QT, Bauchet L, Davis FG, et al. The epidemiology of glioma in adults: a "state of the science" review. Neuro-Oncology. 2014;16(7):896-913

11. Baldi I, Huchet A, Bauchet L, Loiseau H. Épidémiologie des glioblastomes. Neurochirurgie. 2010;56(6):433-440

12. Cungi C, Bouhana S, Degoul G, Bibollet D. Deux échelles brèves d'auto-évaluation des stresseurs et du stress. :33

13. Dhabhar FS. Effects of stress on immune function: the good, the bad, and the beautiful. Immunol Res. 2014;58(2-3):193-210

14. McEWEN BS. Stress, Adaptation, and Disease: Allostasis and Allostatic Load. Annals of the New York Academy of Sciences. 1998;840(1):33-44

15. Hammen C. Stress and Depression. Annu Rev Clin Psychol. 2005;1(1):293-319

16. Steptoe A, Kivimäki M. Stress and cardiovascular disease. Nat Rev Cardiol. 2012;9(6):360-370

17. Kimyai-Asadi A, Usman A. The Role of Psychological Stress in Skin Disease. J Cutan Med Surg. 2001;5(2):140-145

18. Schoultz M, Beattie M, Gorely T, Leung J. Assessment of causal link between psychological factors and symptom exacerbation in inflammatory bowel disease: a systematic review utilising Bradford Hill criteria and meta-analysis of prospective cohort studies. Syst Rev. 2020;9(1):169

19. Epel ES, Crosswell AD, Mayer SE, et al. More than a feeling: A unified view of stress measurement for population science. Frontiers in Neuroendocrinology. 2018;49:146- 169

20. Lantz PM, House JS, Mero RP, Williams DR. Stress, Life Events, and Socioeconomic Disparities in Health: Results from the Americans' Changing Lives Study. J Health Soc Behav. 2005;46(3):274-288

21. Antoni MH, Dhabhar FS. The impact of psychosocial stress and stress management on immune responses in patients with cancer. Cancer. 2019;125(9):1417-1431 
22. Antoni MH, Lutgendorf SK, Cole SW, et al. The influence of bio-behavioural factors on tumour biology: pathways and mechanisms. Nat Rev Cancer. 2006;6(3):240-248

23. Soygur H, Palaoglu O, Akarsu ES, et al. Interleukin-6 levels and HPA axis activation in breast cancer patients with major depressive disorder. Progress in NeuroPsychopharmacology and Biological Psychiatry. 2007;31(6):1242-1247

24. Lu H. Inflammation, a Key Event in Cancer Development. Molecular Cancer Research. 2006;4(4):221233

25. Sloan EK, Priceman SJ, Cox BF, et al. The Sympathetic Nervous System Induces a Metastatic Switch in Primary Breast Cancer. Cancer Research. 2010;70(18):7042-7052

26. Antonova L, Aronson K, Mueller CR. Stress and breast cancer: from epidemiology to molecular biology. Breast Cancer Res. 2011;13(2):208

27. Duijts SFA, Zeegers MPA, Borne BV. The association between stressful life events and breast cancer risk: A meta-analysis. Int J Cancer. 2003;107(6):1023-1029

28. Nielsen NR, Zhang Z-F, Kristensen TS, Netterstr m B, Schnohr P, Gr nbæk M. Self reported stress and risk of breast cancer: prospective cohort study. BMJ. 2005;331(7516):548

29. Pouchieu C, Gruber A, Berteaud E, et al. Increasing incidence of central nervous system (CNS) tumors (2000-2012): findings from a population based registry in Gironde (France). BMC Cancer. 2018;18(1):653

30. Bunevicius A, Deltuva VP, Tamasauskas A. Association of pre-operative depressive and anxiety symptoms with five-year survival of glioma and meningioma patients: a prospective cohort study. Oncotarget. 2017;8(34):57543-57551

31. Esteller M, Garcia-Foncillas J, Andion E, et al. Inactivation of the DNA-Repair Gene MGMT and the Clinical Response of Gliomas to Alkylating Agents. N Engl J Med. 2000;343(19):1350-1354

32. Rozanski A, Blumenthal JA, Kaplan J. Impact of Psychological Factors on the Pathogenesis of Cardiovascular Disease and Implications for Therapy. Circulation. 1999;99(16):2192-2217

33. Kershaw KN, Brenes GA, Charles LE, et al. Associations of Stressful Life Events and Social Strain With Incident Cardiovascular Disease in the Women's Health Initiative. JAHA. 2014;3(3)

34. Fond G, Lancon C, Auquier P, Boyer L. [Prevalence of major depression in France in the general population and in specific populations from 2000 to 2018: A systematic review of the literature]. Presse Med.2019;48(4):365-375

35. Chierichetti SM, Moise G, Galeone M, Fiorella G, Lazzari R. Beta-blockers and psychic stress: a double-blind, placebo-controlled study of bopindolol vs lorazepam and butalbital in surgical patients. Int J Clin Pharmacol Ther Toxicol. 1985;23(9):510-514

36. Steptoe A, Ronaldson A, Kostich K, Lazzarino Al, Urbanova L, Carvalho LA. The effect of betaadrenergic blockade on inflammatory and cardiovascular responses to acute mental stress. Brain Behav Immun. 2018;70:369-375 
37. Andrade C. $\beta$-Blockers and the Risk of New-Onset Depression: Meta-analysis Reassures, but the Jury Is Still Out. J Clin Psychiatry. 2021;82(3):21f14095

38. Riemer TG, Villagomez Fuentes LE, Algharably EAE, et al. Do $\beta$-Blockers Cause Depression?: Systematic Review and Meta-Analysis of Psychiatric Adverse Events During $\beta$-Blocker Therapy. Hypertension. 2021;77(5):1539-1548

39. Bejan-Angoulvant T, Angoulvant D. [Update on beta blockers in 2020]. Rev Med Interne. 2020;41(11):741747

40. Larjavaara S, Mäntylä R, Salminen T, et al. Incidence of gliomas by anatomic location. Neuro Oncol.2007;9(3):319-325

41. Lipsey JR, Robinson RG, Pearlson GD, Rao K, Price TR. Mood Change following Bilateral Hemisphere Brain Injury. Br J Psychiatry. 1983;143(3):266-273

42. Vesterlund GK, Høeg BL, Johansen C, Heitmann BL, E. Bidstrup P. Prolonged job strain and subsequent risk of cancer in women - a longitudinal study, based on the Danish Nurse Cohort. Acta Oncologica. 2017;56(2):301-306.

43. Heikkila K, Nyberg ST, Theorell T, et al. Work stress and risk of cancer: meta-analysis of 5700 incident cancer events in 116000 European men and women. BMJ. 2013;346(feb07 1):f165-f165

44. Stupp R, Hegi ME, Mason WP, et al. Effects of radiotherapy with concomitant and adjuvant temozolomide versus radiotherapy alone on survival in glioblastoma in a randomised phase III study: 5-year analysis of the EORTC-NCIC trial. The Lancet Oncology. 2009;10(5):459-466

45. Crosswell AD, Lockwood KG. Best practices for stress measurement: How to measure psychological stress in health research. Health Psychology Open. 2020;7(2):205510292093307

46. Slavich GM, Shields GS. Assessing Lifetime Stress Exposure Using the Stress and Adversity Inventory for Adults (Adult STRAIN): An Overview and Initial Validation. Psychosom Med. 2018;80(1):17-27

47. Holmes $\mathrm{TH}$, Rahe RH. The social readjustment rating scale. Journal of Psychosomatic Research. 1967;11(2):213-218

48. Lee E-H. Review of the psychometric evidence of the perceived stress scale. Asian Nurs Res (Korean Soc Nurs Sci). 2012;6(4):121-127

49. Oh PJ, Shin SR, Ahn HS, Kim HJ. Meta-analysis of psychosocial interventions on survival time in patients with cancer. Psychology \& Health. 2016;31(4):396-419

50. Phillips KM, Antoni MH, Lechner SC, et al. Stress Management Intervention Reduces Serum Cortisol and Increases Relaxation During Treatment for Nonmetastatic Breast Cancer: Psychosomatic Medicine. 2008;70(9):1044-1049

\section{Tables}

Table 1. Patients characteristics in the study population of IDH wild type glioblastoma patients 
High stressors High stressors

/

High stress

Number of patients

Median age at diagnosis

(years)

Depression history

Psychotropic medication

Beta-blocker medication

Workers

Symptoms at diagnostic :

- Deficit

- Cognitive impairement

- Epilepsy

-Intracranial hpertension

PS $0 / 1$

Tumour location :

- Frontal

- Temporal

- Parietal

- Occipital

- Corpus callosum

- Thalamus

- Multifocal

MGMT methylation

\section{Median Time (month)}

- Acute stress - diagnosis

- First symptom - diagnosis
13 (20,3\%)

58,5

$$
\mathrm{N}=8(61,5 \%)
$$

$\mathrm{N}=4(50 \%)$

$\mathrm{N}=4(11,1 \%)$

$\mathrm{N}=1(7,7 \%) \quad \mathrm{N}=1(12,5 \%)$

$\mathrm{N}=6(16,7 \%)$

$\mathrm{N}=1(7,7 \%) \quad \mathrm{N}=1(12,5 \%)$

$\mathrm{N}=7(19,4 \%)$

$\mathrm{N}=16$

$(44,4 \%)$

\begin{tabular}{|c|c|}
\hline$J=10(76,9 \%)$ & $\mathrm{N}=4(50 \%)$ \\
\hline
\end{tabular}

$N=3(42,9 \%)$

$\mathrm{N}=4(57,1 \%)$

Low stressors

High stress

$7(10,9 \%)$

66,7

$\mathrm{N}=2(28,6 \%)$

$N=1(14,3 \%)$
$\mathrm{N}=5(38,5 \%) \quad \mathrm{N}=4(50 \%) \quad \mathrm{N}=20$

$(55,6 \%)$

$\mathrm{N}=9(69,2 \%) \quad \mathrm{N}=4(50 \%)$

$\mathrm{N}=5(38,5 \%) \quad \mathrm{N}=4(50 \%)$

$\mathrm{N}=16$

$(44,4 \%)$

$\mathrm{N}=1(12,5 \%)$

$\mathrm{N}=14$

$(38,9 \%)$

$\mathrm{N}=1(2,8 \%)$

$N=8(61,5 \%) \quad N=7(87,5 \%)$

$\mathrm{N}=25$

$(69,4 \%)$

$N=5(71,4 \%)$
$\mathrm{N}=2(28,6 \%)$

$\mathrm{N}=3(42,3 \%)$

$N=1(14,3 \%)$ 


\section{Appendix}

Appendix 1 is not available with this version

\section{Figures}

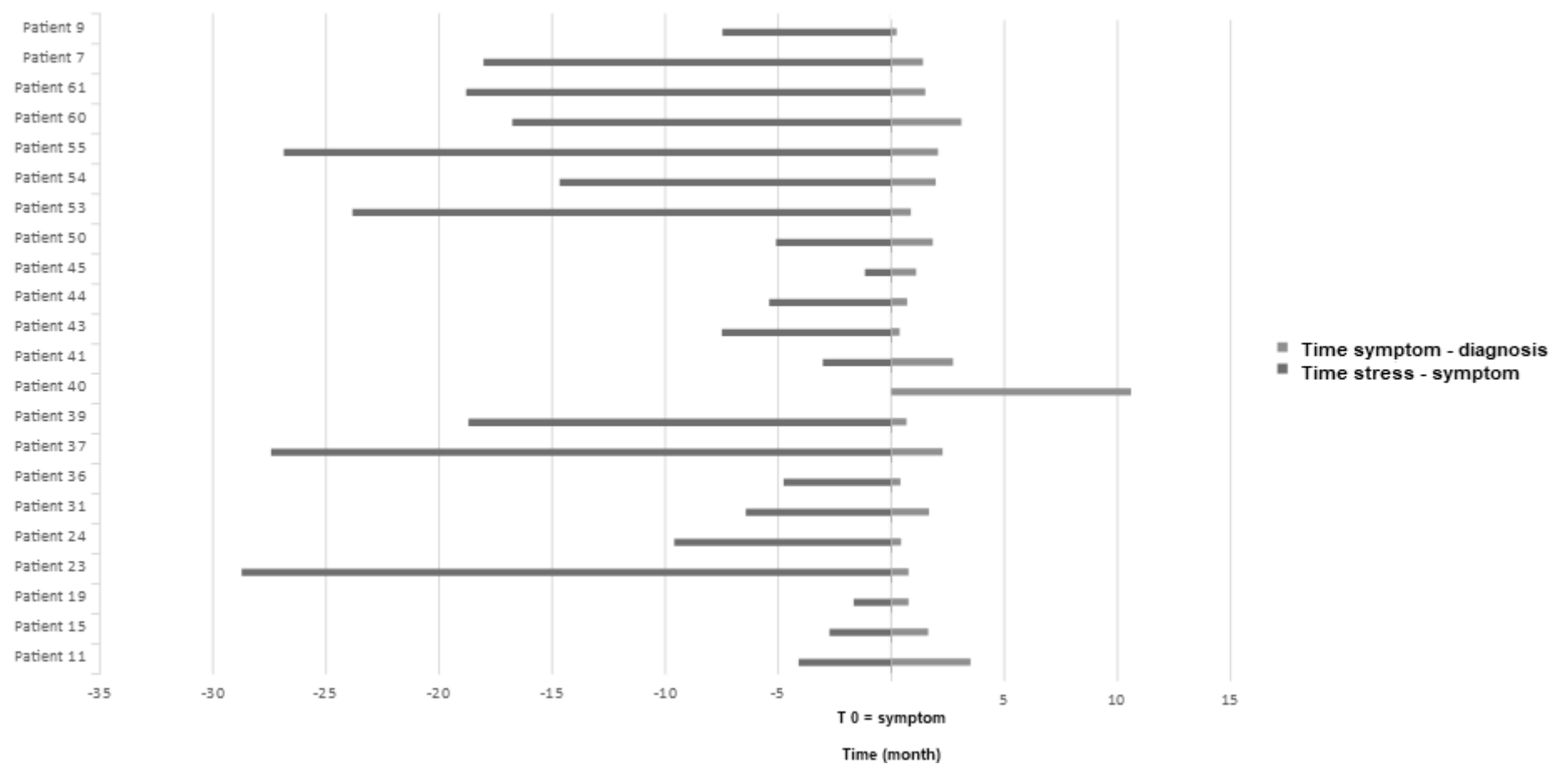

\section{Figure 1}

Time between stress - symptom - glioblastoma diagnosis in patients with acute stress. 0 represented glioblastoma first symptoms, negative part represented time from stress event to first symptoms, and positive part represented time from first symptoms to glioblastoma diagnosis. 